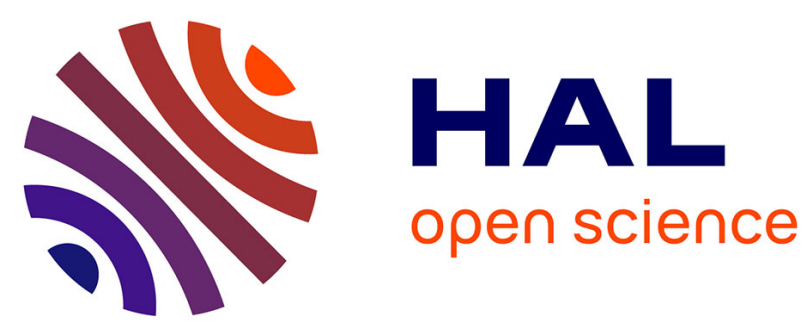

\title{
On the FEM Modal Approach for a Reverberation Chamber Analysis
}

Gerard Orjubin, Elodie Richalot, Stéphanie Mengué, Wong Man-Fai, Odile

Picon

\section{- To cite this version:}

Gerard Orjubin, Elodie Richalot, Stéphanie Mengué, Wong Man-Fai, Odile Picon. On the FEM Modal Approach for a Reverberation Chamber Analysis. IEEE Transactions on Electromagnetic Compatibility, 2007, 49 (1), pp.76-84. 10.1109/TEMC.2006.888187 . hal-01441548

\author{
HAL Id: hal-01441548 \\ https://hal.science/hal-01441548
}

Submitted on 19 Jan 2017

HAL is a multi-disciplinary open access archive for the deposit and dissemination of scientific research documents, whether they are published or not. The documents may come from teaching and research institutions in France or abroad, or from public or private research centers.
L'archive ouverte pluridisciplinaire HAL, est destinée au dépôt et à la diffusion de documents scientifiques de niveau recherche, publiés ou non, émanant des établissements d'enseignement et de recherche français ou étrangers, des laboratoires publics ou privés. 


\title{
On the FEM Modal Approach for Reverberation Chamber Analysis
}

\author{
Gérard Orjubin $^{1}$, Elodie Richalot ${ }^{1}$, Member, IEEE, Stéphanie Mengué ${ }^{1}$, Man-Fai Wong, Member, \\ IEEE, and Odile Picon ${ }^{1}$, Member, IEEE
}

\begin{abstract}
We review the difficulties of a modal approach when modeling a Reverberation Chamber (RC) by the Finite Element Method (FEM). The numerical challenge is due to the large scale problem involved by the over-dimensioned cavity. Moreover, the field singularity on the stirrer has to be captured by the FEM. First the following issues are discussed: existence of nullfrequency solutions, convergence rate for $h$ and $p$ adaption, and formulation type in $\mathrm{E}$ or $\mathrm{H}$ field. Then the modal analysis is compared to the classical harmonic one. A focus is put on the field singularity at the source point.
\end{abstract}

Index Terms-Cavity, electromagnetic analysis, finite element methods, large-scale eigenproblem, reverberation chamber.

\section{INTRODUCTION}

$\mathrm{T}$ he Reverberation Chamber (RC) is a useful tool employed for Electromagnetic Compatibility measurement. It consists of a closed metallic cavity containing a metallic stirrer. High field levels can be generated in this low loss enclosure using modest source power, and allow susceptibility tests on electronic devices. The field within a cavity being essentially composed of standing waves, the moving metallic stirrer contributes to randomize the field. At high frequencies, the field can be statistically uniform and isotropic over a working volume, for a sufficient perturbation induced by a stirrer displacement, generally a rotation. This statistical approach has been validated experimentally for high frequencies [1].

As illustrated by Bruns and Vahldieck in [2], modeling is helpful to better understand the RC stirring process at low frequencies. This author gives a complete overview of the different techniques already used, most of them being based on a point by point harmonic approach. As an exception, the modal analysis of the RC was first investigated by Bunting in [3] for the 2D case, the author pointing out the numerical difficulties. Since this paper publication (1999), many improvements have been made in term of software and hardware: the main purpose of the present paper is to show how state-of-the-art techniques allow a resolution of the $3 \mathrm{D}$ problem on a simple PC.

Manuscript received November 15, 2005

The authors ${ }^{1}$ are with the laboratoire ESYCOM, Universite de Marne la Vallée, France (orjubin@ univ-mlv.fr).

M. F. Wong is with France Telecom R\&D, Issy les Moulineaux, France.
The attention must be drawn on the fact that we deliberately restrict the study to a very basic RC, despite the importance of small geometric details, such as RC doors, as illustrated in [2]. As another restriction of our study, the frequency range concerns the first modes of the RC. Although these facts deserve a special attention, our previous work [4] showed interesting properties of the modal technique, such as a possibility to study the influence of the quality factor on the RC stirring process, and a capability of analyzing the modes perturbation induced by the stirrer rotation. In fact, the present paper is dedicated to the fundamental and the numerical aspects of the modal method.

Mode determination is used for other applications than RC: for instance the modeling of dielectric resonator by the Finite Integral Technique (FIT) [5] or the analysis of particle accelerator cavity by the Finite Element Method (FEM) [6]. We used this latter modeling method implemented in the Open Source Pyfemax program [24] and in commercial Femlab® V3.0 software.

The lossless RC eigenmodes are directly determined by the Maxwell equations discretization that leads to a large scale eigenproblem.

We first compare the performance of the solvers Implicitly Restarted Arnoldi Method (IRAM) and Jacobi-Davidson (JD). Then a specific problem arising with FEM is exposed, i.e. the existence of null-frequency modes. A Tree-Cotree technique [7] has been proposed to discard these modes, using a topological analysis of the discretization mesh. We check in Section II that the technique used in [6] also finds its origin in a topological matrix. Then a numerical example shows the reduction of the CPU time.

In Section III, the $h$ and $p$ adaption issues are investigated. As the field may be singular on stirrer edges, the consequences of these singularities on the convergence rate are presented in Section IV.

The excitation of this enclosure creates another field singularity at the source point. Its contribution to the modal expansion is examined and a regularization technique is implemented in Section V.

\section{MODE DETERMINATION}

The basic geometric model of the RC used in this study is depicted in Fig. 1: 


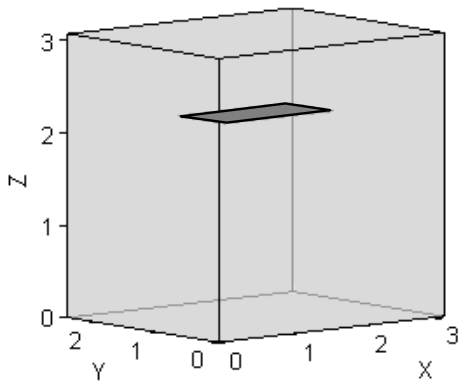

Fig. 1. RC with its rectangular metallic stirrer.

A planar metallic stirrer $(1.5 \mathrm{~m} \times 0.75 \mathrm{~m})$ is placed in a $(3.10 \mathrm{~m} \times 2.47 \mathrm{~m} \times 3.07 \mathrm{~m})$ metallic rectangular cavity. The stirrer is centered, placed at $0.87 \mathrm{~m}$ below the roof and parallel to it. Only one stirrer position is analysed, for which the stirrer edges are parallel to the cavity walls. In the main part of this text, results are presented for the frequency band 50 100 $\mathrm{MHz}$, which contains 5 eigenmodes when considering the empty stirrerless cavity. For the eigenmode analysis (Sections II IV), the antenna modeling is useless.

\section{A. Large scale eigenvalue problem}

This lossless problem verifies the double rotational equation for the complex $\boldsymbol{E}$ field:

$$
\left\{\begin{array}{ll}
R \vec{o} t \operatorname{Ro} t \vec{E}=\left(\frac{\omega}{c}\right)^{2} \vec{E}=\lambda \vec{E} & \text { in } \Omega \\
\vec{n} \wedge \vec{E}=\overrightarrow{0} & \text { on } \partial \Omega
\end{array} .\right.
$$

where $\Omega$ designates the cavity inside.

Projecting the field on $N$ global edge elements $\vec{w}_{k}$, are defined $N$ Degrees of Freedom (DoF) $\vec{E} \cong \sum_{1}^{N} e_{k} \vec{w}_{k}$, and a vector $e$ of DoF. These elements are named global, as they are not related to a specific tetrahedron. Then, the Galerkin method yields the algebraic equation

$$
\mathbf{R} \vec{e}=\lambda \mathbf{M} \vec{e}
$$

where the stiffness $\mathbf{R}$ and mass $\mathbf{M}$ matrices are defined by:

$$
\left\{\begin{array}{l}
r_{j k}=\iiint_{\Omega} R \vec{o} t \vec{w}_{j} \cdot R \vec{o} t \vec{w}_{k} d V \\
m_{j k}=\iiint_{\Omega} \vec{w}_{j} \cdot \vec{w}_{k} d V
\end{array} .\right.
$$

This generalized eigenproblem leads to a direct determination of modes: each eigenvalue is linked to a resonant frequency, whereas the eigenvector corresponds to the mode cartography. $\mathbf{R}$ and $\mathbf{M}$ are real symmetric matrices whose dimension (number of DoF) is high, say $N=10^{5}$. In such a case, specific techniques exist to solve this large eigenproblem.

Based on a projected solution space, Implicitly Restarted Arnoldi Method (IRAM), Implicitly Restarted Lanczos (IRL) and Jacobi-Davidson (JD) algorithms are suited to the solving of (2), particularly because $\mathbf{R}$ and $\mathbf{M}$ are sparse matrices.
Looking for a small set of eigenmodes, one has to specify the eigenvalue target $\sigma$ and the number of modes sought. In our case, 6 eigenmodes are sought close to the target $\sigma=2$.

Femlab® features an IRAM solver, which is associated to a Shift-and-Invert spectral transformation: a factorization of the real symmetric matrix $\mathbf{R}-\sigma \mathbf{M}$ is therefore necessary [6], for which several factorization algorithms are available on Femlab software. After numerical tests, the Spooles factorization algorithm is retained, the performance of this direct method being such that the iterative techniques are useless.

On another hand, JD is a factorization-free algorithm, implemented in Pyfemax [8][22]. That program also includes hierarchical second order $\mathbf{H}(\mathbf{R o t})$-conform elements and a powerful Algebraic MultiGrid (AMG) technique.

The comparison of these two solvers is given in Fig. 2, for finding the $6 \mathrm{RC}$ modes present in the $50 \sim 100 \mathrm{MHz}$ band.

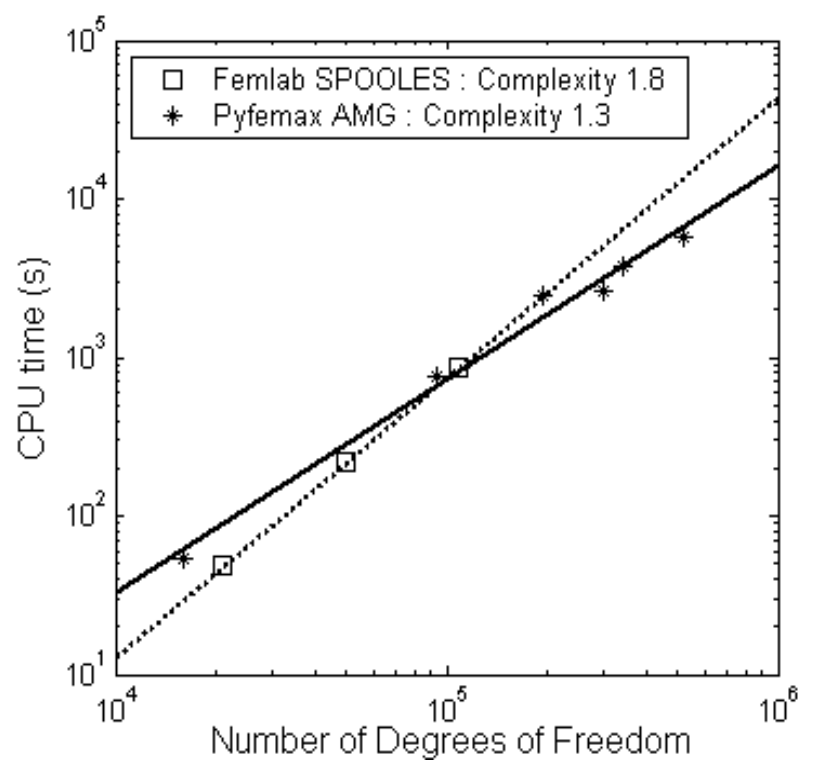

Fig. 2. Computation time for determining the 6 modes present in the band 50 to $100 \mathrm{MHz}$. With Femlab, first order edge elements are used, and the solving is performed using IRAM solver and the Spoole factorization algorithm. With Pyfemax, hierarchical second order elements are used with an AMG technique, and the solving involves a JD algorithm.

The first conclusion is about the CPU time: $10^{3} \mathrm{~s}$ for $10^{5} \mathrm{DoF}$ is fairly good when the platform is a mere $1.2 \mathrm{GHz}$ PC. The second observation is that the algorithmic complexity makes the MultiGrid technique a must for larger problems, with a complexity of 1.3 to be compared to the complexity of 1.8 obtained with Femlab.

\section{B. Structure of the discrete solution space}

Taking the divergence of (1) leads to

$$
\left(\frac{\omega}{c}\right)^{2} \operatorname{Div}(\vec{E})=0 .
$$

Non-null-frequency modes are solenoidal (null-divergence): more generally, the solutions can be either solenoidal $\left(\boldsymbol{E}_{\text {sol }}\right)$ either null-frequency irrotationnal $\left(\boldsymbol{E}_{i r r}\right)$. These two kinds of 
solutions are orthogonal in the following sense:

$$
\vec{E}_{i r r} \perp \vec{E}_{\text {sol }} \Leftrightarrow\left(\vec{E}_{i r r}, \vec{E}_{s o l}\right)=0 \Leftrightarrow \iiint_{\Omega} \vec{E}_{i r r} . \vec{E}_{\text {sol }} d V=0
$$

The solution space $\mathbf{H}_{\mathbf{0}}$ (Rot) can be split in two infinite dimension spaces, a solenoidal and an irrotational space, as indicates the Helmholtz decomposition:

$$
\begin{array}{ll}
\qquad \mathbf{H}_{\mathbf{0}}(\mathbf{R o t})=\mathbf{W}_{\text {sol }} \oplus \mathbf{G r a d H}_{\mathbf{0}} \\
\text { where } \quad \mathbf{W}_{\text {sol }}=\left\{\boldsymbol{u} \in \mathbf{H}_{\mathbf{0}}(\mathbf{R o t}) / \operatorname{Div}(\boldsymbol{u})=0\right\} \\
& \mathbf{G r a d H}_{\mathbf{0}}=\left\{\boldsymbol{u} \in \mathbf{H}_{\mathbf{0}}(\operatorname{Rot}) / \operatorname{Rot}(\boldsymbol{u})=\mathbf{0}\right\} .
\end{array}
$$

When using the so called edge elements introduced by Nédélec [9], the splitting is respected in a discrete sense: to get an idea about this fact, we recall some properties of these elements. If $L_{i}$ and $L_{j}$ are the Lagrange scalar functions associated to $i$ and $j$ points of a given tetrahedron, then the edge element is

$$
\vec{w}_{i j}=L_{i} G \overrightarrow{r a} d L_{j}-L_{j} G \overrightarrow{r a} d L_{i} .
$$

These elements (named local as they are associated to a given tetrahedron) verify $\operatorname{Div}\left(\boldsymbol{w}_{i j}\right)=0$, whereas the global elements $\boldsymbol{w}_{k}$ permit to describe correctly the non-null-divergence solutions $\boldsymbol{E}_{\text {sol }}$. On the other hand, we have

$$
-\left(\vec{w}_{12}+\vec{w}_{13}+\vec{w}_{14}\right)=G \vec{a} d L_{1}
$$

This means that $\operatorname{Grad}\left(L_{l}\right)$ is an algebraic sum of the edge elements linked to node 1. Equation (7), concerning local elements $L_{j}$ and $\boldsymbol{w}_{i j}$, can be generalized to elements $\varphi_{j}$ and $\boldsymbol{w}_{k}$ :

$$
\operatorname{Gra} d \varphi_{j}=\sum_{k=1}^{N} g_{k j} \vec{w}_{k} \quad \forall j \in\left[\begin{array}{ll}
1 & P
\end{array}\right]
$$

where $N$ is the edge number and $P$ the node number.

The $\left(g_{k j}\right)$ permit to define a topological sparse matrix $\mathbf{G}$ that can be considered as a discretized gradient. If $\vec{v}=\left(v_{1}, v_{2}, \ldots, v_{N}\right)$ stands for $V=\sum_{i=1}^{P} v_{i} \varphi_{i}$, then $\mathbf{G} \vec{v}$ corresponds to

$$
\sum_{k=1}^{N} \sum_{i=1}^{P} g_{k i} v_{i} \vec{w}_{k}=\sum_{i=1}^{P} \sum_{k=1}^{N} g_{k i} v_{i} \vec{w}_{k}=\sum_{i=1}^{P} v_{i} \operatorname{Gr} \vec{a} d \varphi_{i} .
$$

More properties can be presented: if $\Phi_{\mathrm{h}}$ is the scalar space of the nodal functions, (8) means that $\operatorname{Grad}\left(\Phi_{\mathrm{h}}\right)$ is included in the vectorial space $\mathbf{W}_{\mathbf{h}}$ of Nédélec functions. In fact, this inclusion property can be extended to a discrete decomposition [6]:

$$
\mathbf{W}_{\mathbf{h}}=\mathbf{W}_{\mathbf{h ~ s o l}} \oplus \operatorname{Grad}\left(\Phi_{\mathrm{h}}\right) .
$$

Because of $\mathbf{R}$ definition (2), it is easy to check that

$$
\mathbf{R G}=\mathbf{0} \text {. }
$$

This means that $\operatorname{Grad}\left(\Phi_{\mathrm{h}}\right)$ space corresponds to the $\mathbf{R}$ kernel, i.e. the null-frequency solutions $\boldsymbol{e}_{\boldsymbol{0}}$, as $\mathbf{R} \boldsymbol{e}_{\boldsymbol{0}}-\lambda \mathbf{M} \boldsymbol{e}_{\boldsymbol{0}}=\mathbf{0}$ implies $\lambda \mathbf{M} \boldsymbol{e}_{\boldsymbol{0}}=\mathbf{0}$ or $\lambda=0$ (M being positive defined). Thus $\mathbf{W}_{\text {hsol }}$ is the space of researched non-null-frequency modes.

The relation (11) can be interpreted in the more conceptual way using the differential form spaces $\mathrm{W}^{\mathrm{n}}$, as suggested by Bossavit in 1988 [10]. These spaces respect inclusion relations, that are outlined in the De Rham complex:

$$
\mathrm{W}^{0} \stackrel{\mathrm{G}}{\longrightarrow} \mathrm{W}^{1} \stackrel{\text { Ro }}{\longrightarrow} \mathrm{W}^{2} \stackrel{\mathrm{D}}{\longrightarrow} \mathrm{W}^{3} \text {. }
$$

The differentiation of $\mathrm{W}^{0}$ (resp. $\mathbf{W}^{\mathbf{1}}$ ) via the discrete Grad (Resp. Rot) operator is included in the $\mathbf{W}^{\mathbf{1}}\left(\operatorname{Resp} \mathrm{W}^{2}\right)$ space, and so on. The $\operatorname{Rot}(\mathbf{G r a d})=\mathbf{0}$ relation is similar to (11). In the case of trivial topology, $\mathbf{W}^{\mathbf{1}}$ (i.e. $\mathbf{W}_{\mathbf{h}}$ ) is a sum of two subspaces: $\operatorname{Grad}\left(\mathrm{W}^{0}\right)$ (the kernel of discretised Rot operator) and the complementary space of null-divergence elements, as indicated by (10).

Summing up, the use of Nédélec elements leads to a singular $\mathbf{R}$ matrix, and the $\mathbf{R}$ kernel corresponds to the nullfrequency solutions of (2). Taking into account the frontier conditions, the number of these non physical solutions is equal to the number of mesh interior points and can be estimated [11] as $14 \%$ of the $\mathbf{R}$ dimension, $N$. Because $N$ is as large as $10^{5}$, one understands the need to deal with these solutions.

\section{Elimination of the Null-frequency solutions}

From the definitions of $\mathbf{M}$ (2) and of the field orthogonality (4), one can write

$$
\vec{e}_{i r r}^{T} \mathbf{M} \vec{e}_{s o l}=0
$$

As the null-frequency solutions $\boldsymbol{e}_{\text {irr }}$ belong to the $\mathbf{R}$ kernel, i.e. to the $\mathbf{G}$ image (11), we can write $\boldsymbol{e}_{i r r}=\mathbf{G} \boldsymbol{v}$. The Morthogonality of the solutions of (2) yields:

$$
\begin{gathered}
(\forall \vec{v}) \vec{v}^{T} \mathbf{G}^{\mathbf{T}} \mathbf{M} \vec{e}_{s o l}=0 \Rightarrow(\forall \vec{v}) \vec{v}^{T} \mathbf{C}^{\mathbf{T}} \vec{e}_{s o l}=0 \\
\Rightarrow \mathbf{C}^{\mathbf{T}} \vec{e}_{s o l}=0 \text { with } \mathbf{C}=\mathbf{M G} .
\end{gathered}
$$

Respecting (15), the resonant frequencies will be non-null: condition (15) can be implemented in the solver through various techniques: see [6] for JD or [12] for IRL.

It is interesting to note that (15) can be derived in another way, enforcing $\operatorname{Div} \boldsymbol{E}=0$ with a Lagrange multiplier p. This is Kikuchi method, recalled in [6], that leads to the weak variational statement: find $\boldsymbol{E} \in \mathbf{H}_{\mathbf{0}}$ (Rot) and $p \in \mathrm{H}_{0}{ }^{1}$ such that

$$
\left\{\begin{array}{l}
\left(\operatorname{Ra} t \vec{E}, \operatorname{Ro} t \vec{E}_{1}\right)+\left(\operatorname{Gra} d p, \vec{E}_{1}\right)=\lambda\left(\vec{E}, \vec{E}_{1}\right) . \\
\left(\vec{E}, \text { Gra } d p_{1}\right)=0
\end{array} .\right.
$$

for any test functions $\boldsymbol{E}_{1}$ and $p_{l}$. 
Discretizing $\boldsymbol{E}$ on edge elements (vector $\boldsymbol{e}$ of unknowns) and $p$ on Lagrange elements (vector $\boldsymbol{x}$ ), it comes

$$
\begin{array}{r}
{\left[\begin{array}{ll}
\mathbf{R} & \mathbf{C} \\
\mathbf{C}^{\mathbf{T}} & \mathbf{0}
\end{array}\right]\left[\begin{array}{l}
\mathbf{e} \\
\mathbf{x}
\end{array}\right]=\lambda\left[\begin{array}{cc}
\mathbf{M} & \mathbf{0} \\
\mathbf{0} & \mathbf{0}
\end{array}\right]\left[\begin{array}{l}
\mathbf{e} \\
\mathbf{x}
\end{array}\right]} \\
\text { where } c_{i j}=\left(\vec{w}_{i}, G r \vec{a} d \varphi_{j}\right) .
\end{array}
$$

C is actually the same as given by (15), as shows (18):

$$
c_{i j}=\left(\vec{w}_{i}, \sum_{k} g_{k j} \vec{w}_{k}\right)=\sum_{k} g_{k j}\left(\vec{w}_{i}, \vec{w}_{k}\right)=\sum_{k} g_{k j} m_{i k} .
$$

As $\boldsymbol{x}$ is a Lagrange multiplier, expressing $\operatorname{Div} \boldsymbol{E}=0$ (i.e. nonnull-frequency) leads to the same condition (15). Whatever the method is, (15) permits to eliminate the non-physical modes.

To illustrate the advantage of this restricted research, we used the Pyfemax program that includes an implementation of (15) in the JD direct solver, called SAUG, and compare the computation times with SAUG algorithm and without this option (direct solver). In both cases, the first steps of assembling and conditioning the matrix are unchanged. The computation time (s) for determining the modes in the 50 100 $\mathrm{MHz}$ band are given in Table I (mesh with $1710^{3}$ tetrahedra).

TABLE I

COMPARISON OF PYFEMAX SOLVER OPTION FOR ELIMINATION OF NULLFREQUENCY MODES (CPU TIMES IN S.)

\begin{tabular}{l|l||l}
\cline { 2 - 3 } \multicolumn{1}{c||}{} & $\begin{array}{l}\text { Order 1 elements } \\
210^{4} \mathrm{DoF}\end{array}$ & $\begin{array}{l}\text { Order } 2 \text { elements } \\
1.110^{5} \mathrm{DoF}\end{array}$ \\
\hline \hline Assembling & 11 & 17 \\
\hline Conditionning & SuperLu: 37 & SuperLu: 37 \\
\hline Direct solver & 28 & 405 \\
\hline SAUG & 25 & 352 \\
\hline
\end{tabular}

For both dicretization orders, elimination of irrotational solutions by SAUG algorithm permits to save $10 \%$ off the solver computation time.

\section{NODAL AND EDGE ELEMENTS COMPARISON}

The nodal elements, also called Lagrange elements, are well-known to generate the so-called spurious modes. In fact, these modes appearance is due to the equation discretization. In other words, nodal elements can successfully be used to solve another equation than (1). This is done in this Section.

The solution space structure yields the existence of irrotational solutions (e.g. $\operatorname{Grad} \phi$ ). As the $\operatorname{Grad} \phi$ discretization must be continuous (class $\mathrm{C}^{0}$ ), this implies that the $\phi$ discretization must be of class $C^{1}$. This condition can not be respected by using first order Lagrange elements for the $\phi$ discretization: this is the origin of the spurious solutions, as mentioned in [13, Sec. 7.2.1].

In the next paragraph is illustrated the penalty technique [8] that overcomes this problem: it is then possible to compare the precision of nodal and edge elements, and check that these latter should be preferred, as reported by Bardi et al. in [14]. Furthermore, the numerical advantages of second order elements are examined as an extention of a previous work [15].

\section{A. Penalty method}

This method consists in adding a penalty term to (1):

$$
\begin{cases}\operatorname{Rot} \operatorname{Ro} t \vec{E}-s \text { Gra } d \operatorname{Div} \vec{E}-\lambda \vec{E}=\overrightarrow{0} & \text { in } \Omega \\ \vec{n} \wedge \vec{E}=\overrightarrow{0} \text { and Div } \vec{E}=0 & \text { on } \partial \Omega\end{cases}
$$

Taking the divergence of (19) and defining $e=\operatorname{Div} \boldsymbol{E}$ leads to:

$$
\begin{cases}-\Delta e=\frac{\lambda}{s} e=\mu e & \text { in } \Omega \\ e=0 & \text { on } \partial \Omega\end{cases}
$$

The smallest eigenvalue $\mu_{0}$ of this scalar equation can be determined using the FEM with first order Lagrange elements. If $\mu<\mu_{0}$, the unique solution of (20) is trivial, i.e. $e=\operatorname{Div} \boldsymbol{E}=$ 0 . Thus, the irrotational solutions of (19) are eliminated if the penalty parameter $s$ is taken to be higher than $\lambda / \mu_{0}$.

\section{B. Application to the rectangular stirrerless cavity}

For instance, we found $\mu_{0}=3.8 . \lambda=3.8$ being the higher eigenvalue in the frequency band 50 to $100 \mathrm{MHz}$, it means that $s>1$ guarantees that the modes check $\operatorname{Div} \boldsymbol{E}=0$ and are not spurious. To illustrate this fact, we look for the eigenmodes present in this band, knowing that there are only 5 physical modes in the stirrerless cavity. For various penalty parameter values, the number of found modes is reported in Fig. 3.

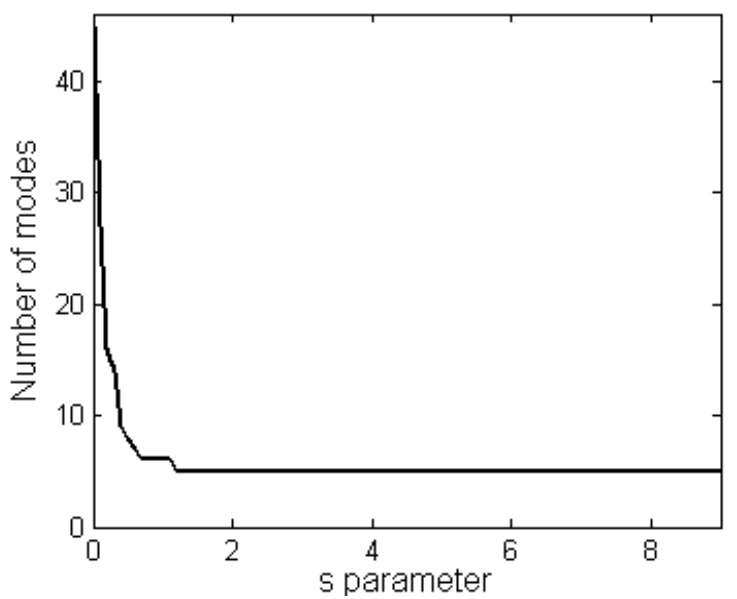

Fig. 3. Influence of the penalty parameter on the number of modes in the 50$100 \mathrm{MHz}$ band for the stirrerless cavity, using first order Lagrange elements.

The spectral pollution by spurious modes is avoided for this frequency band if $s>1$. As a particularity, we note that $s=1$ is a right value for determining the fundamental of a cavity and leads to a closed-form equivalent of (19) as: 


$$
\vec{\Delta} \vec{E}+\lambda \vec{E}=\overrightarrow{0}
$$

An advantage of the study of the stirrerless rectangular cavity is that resonant frequencies are known analytically. This enables a convergence study, for a $h$-adaption scheme, using Femlab (Lagrange elements) and Pyfemax (edge elements) software: the results are presented in Fig. 4.

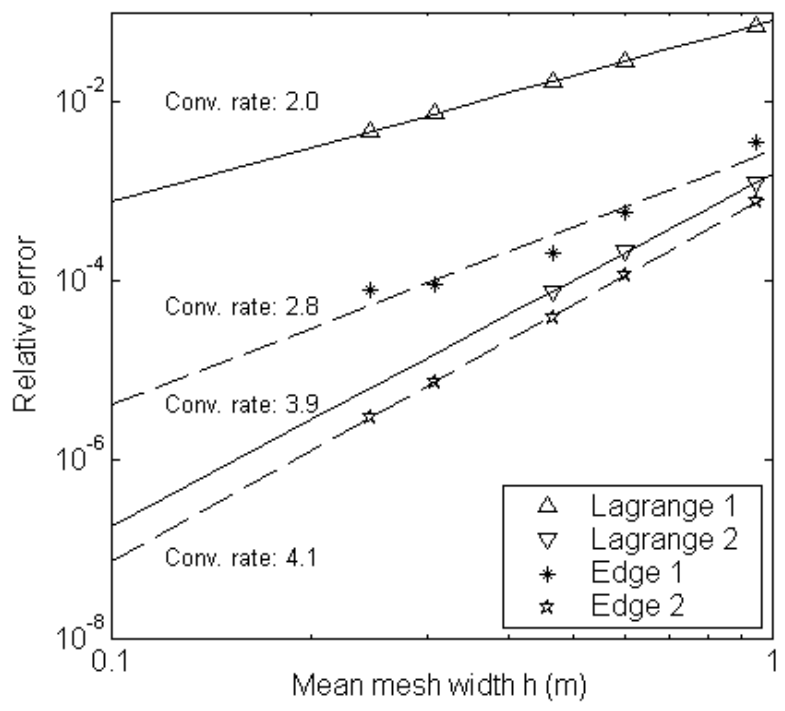

Fig. 4. Mean precision for determining the resonant frequencies of the stirrerless cavity in $50-100 \mathrm{MHz}$ band.

The nodal element convergence rate fits to the theory: the error over the eigenvalue determination is [13, Sec. 2.5.3]:

$$
\left|\lambda_{\text {Exact }}-\lambda_{\text {Numerical }}\right| \propto h^{2 \operatorname{Min}(a, p)}
$$

where $p$ is the element order, $a$ is the regularity coefficient and $h$ the mean mesh width. As the field inside the rectangular cavity is regular, the convergence rate is $2 p$. An important point is the good performance of the H(Rot)-conform (both first and second order edge elements) in term of convergence rate, and in term of numerical properties, as the $\mathbf{R}$ matrix is sparser with edge elements.

However, these results would be far different when modeling the RC. Firstly, it has been shown that the penalty method must be modified in case of re-entrant corner [16]. Actually, the use of the simplistic (19) for a cavity including a stirrer gives no trustable result. A second remark concerns the regularity of the electric field: the stirrer creates a field singularity, that limits the convergence rate seen in (22) (as the regularity $a$ is low). When extending this property to $\mathbf{H}(\mathbf{R o t})$ conform elements, it comes that the convergence is slower if the cavity contains a stirrer. This is investigated in Section IV.

\section{FIELD SINGULARITY ISSUES}

\section{A. $h$ and $p$ adaption}

As already seen, the penalty method (19) can not be employed to model the cavity with the stirrer. This one strongly modifies the field and a high gradient may exist. For instance, the first eigenvector cartography, portrayed in Fig. 5, shows high edge effects at the stirrer vicinity.

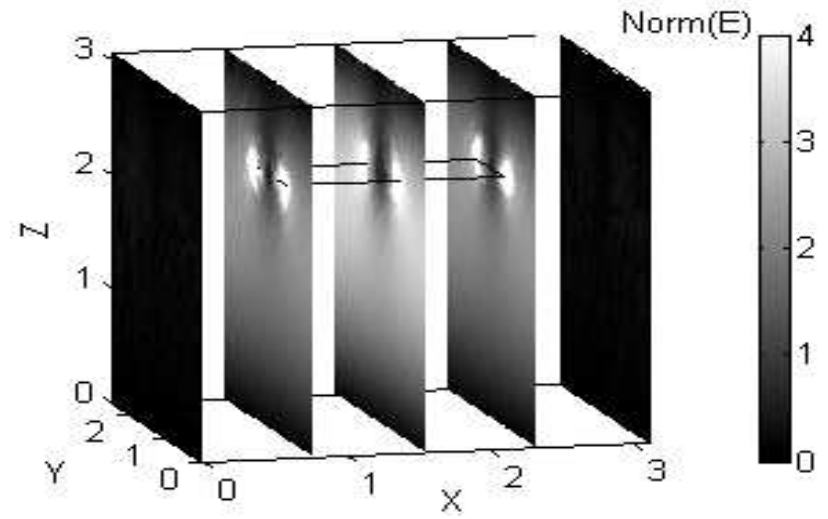

Fig. 5. Cartography of the mode 1 of the RC.

Figure 6 represents the first resonant frequency obtained with Pyfemax for various meshes, using $\mathbf{H}(\mathbf{R o t})$-conform elements of order 1 (Nédélec) and order 2:

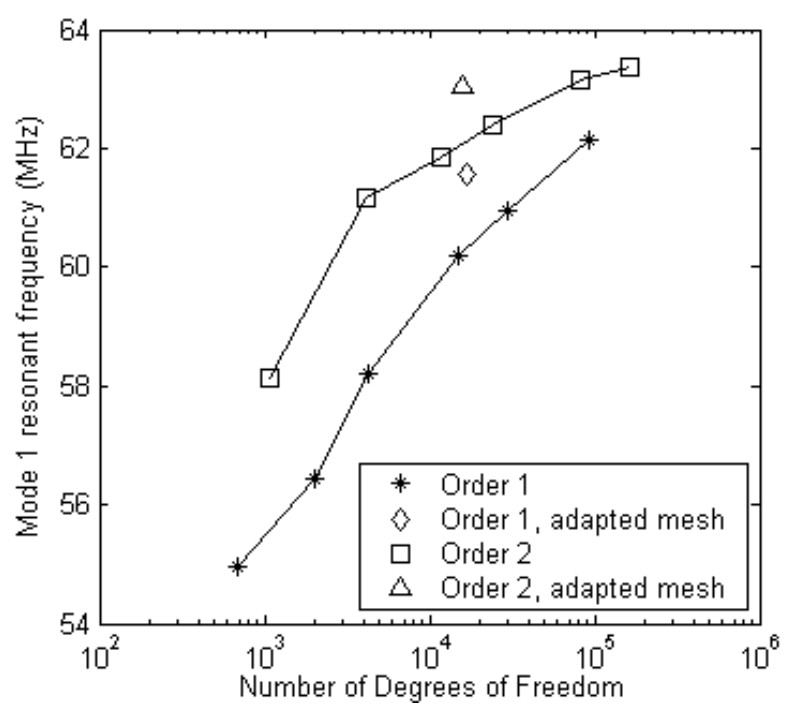

Fig. 6. Convergence study of the RC first mode, using H(Rot)-conform elements.

The monotonous convergence illustrates the advantage of order 2 over order 1 ( $p$-adaption). It also proves the benefit of a mesh finely refined close to the stirrer, called "adapted mesh" in Fig. 6. Note that this a priori non-uniform mesh is not stricto sensu an adapted mesh to a given mode. In fact $h p$ adaption technique is suited to driven frequency analysis or to the determination of a single eigenmode, but not to our study of a set of eigenmodes. 
Another remark must be done concerning the modeling of singularities: instead of higher order regular (polynomial) elements, it seems advantageous to use singular elements at a lower order [17].

\section{B. $E$ and $H$ formulation}

Some interesting conclusions may be drawn from the theory of singularity. In the case of a plane wave diffraction by a infinite plane, Van Bladel [18] has shown that the $\boldsymbol{E}$ field components are equivalent to

$$
\left\{\begin{array}{l}
\left|E_{Z}\right| \propto r^{v} \\
\left|E_{T}\right| \propto r^{\nu-1}
\end{array} \quad 0<v<1,\right.
$$

where $E_{Z}$ and $E_{T}$ are respectively the components along the edge direction and transverse, $r$ is the distance to the edge and $v$ is the singularity exponent. In case of diffraction on a flat sector, the author gives a comparison (Fig. 7) between the singularity exponent $\boldsymbol{v}$ (for $\boldsymbol{E}$ ) and $\tau$ (for $\boldsymbol{H})$ :
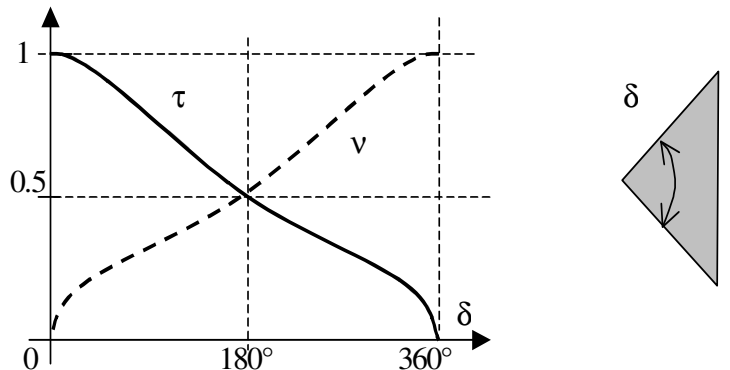

Fig. 7. $\boldsymbol{E}$ and $\boldsymbol{H}$ singularity exponents for a flat sector, from [18, Fig. 5.17].

For a convex corner angle, the $\boldsymbol{H}$ singularity is better than the $\boldsymbol{E}$ singularity. This property of the diffracted waves seems to be valid for the study ${ }^{1}$ of modes, as shows Table II in the case of the RC:

TABLE II

FORMULATION INCIDENCE ON THE RESONANT FREQUENCIES (MHZ) : 6 RC MODES PRESENT IN 50-100 MHZ BAND

\begin{tabular}{l|l|l||l|l|l}
\hline \multicolumn{3}{c||}{ E Formulation } & \multicolumn{3}{c}{ H Formulation } \\
\hline Mesh1 & Mesh2 & Mesh3 & Mesh1 & Mesh2 & Mesh3 \\
5.5 & 1.0 & 2.0 & 8.1 & 1.4 & 2.2 \\
$10^{3}$ & $10^{4}$ & $10^{5}$ & $10^{3}$ & $10^{4}$ & $10^{5}$ \\
DoF & DoF & DoF & DoF & DoF & DoF \\
\hline \hline 60.406 & 61.357 & 63.065 & 63.882 & 63.719 & 63.295 \\
\hline 64.884 & 65.131 & 65.816 & 66.220 & 66.191 & 65.935 \\
\hline 76.974 & 77.221 & 77.274 & 77.125 & 77.282 & 77.281 \\
\hline 90.228 & 90.312 & 90.517 & 90.197 & 90.595 & 90.563 \\
\hline 91.690 & 91.409 & 91.441 & 90.967 & 91.398 & 91.450 \\
\hline 92.511 & 93.120 & 93.715 & 93.533 & 93.637 & 93.753 \\
\hline
\end{tabular}

\footnotetext{
${ }^{1}$ The $\mathrm{H}$ formulation implies a 3D (thick) stirrer modeling. A parallelipedic volume is taken $\left(\delta=90^{\circ}\right)$.
}

The first conclusion is that the mesh has to be farely refined to get close results for $\mathrm{E}$ and $\mathrm{H}$ formulations, as Mesh3 corresponds to more than $210^{5}$ DoF. Furthermore, this convergence study indicates a clear precision enhancement for the $\mathrm{H}$ formulation. Although these results only correspond to the 6 first modes of our $\mathrm{RC}$, it is worth noting that it is consistent with theoretical aspects outlined in Fig. 7.

\section{COMPARISON TO HARMONIC ANALYSIS}

Once the modes are determined, it becomes possible, in a single post-treatment, to get the spectrum of the field when the $\mathrm{RC}$ is excited by an antenna. A comparison with a direct harmonic modeling is presented in this section.

\section{A. Modal expansion}

For an enclosure excited by a harmonic driven current $\boldsymbol{J}$ of pulsation $\omega$, (1) becomes

$$
\left\{\begin{array}{ll}
\operatorname{Ro} t \operatorname{Ro} t \vec{E}=-j \omega \mu_{0} \vec{J}+\left(\frac{\omega}{c}\right)^{2} \vec{E} & \text { in } \Omega \\
\vec{n} \wedge \vec{E}=\overrightarrow{0} & \text { on } \partial \Omega
\end{array} .\right.
$$

The general solution of (24) is expanded as follows [19, §2.3]:

$$
\vec{E}=\sum_{n=1}^{\infty} \alpha_{n} \vec{E}_{n}^{s o l}+\sum_{n=1}^{\infty} \beta_{n} \vec{E}_{n}^{i r r}+\sum_{n=1}^{M-1} \delta_{n} \vec{E}_{n}^{\text {stat }}
$$

where $\boldsymbol{E}_{n}{ }^{\text {sol }}$ are the solenoidal solutions of (1), $\boldsymbol{E}_{n}{ }^{\text {stat }}$ are the static solutions due to the RC topology ${ }^{2}$ and $\boldsymbol{E}_{\boldsymbol{n}}{ }^{i r r}$ are the gradient of solutions of Dirichlet problem:

$$
\begin{cases}\Delta \phi=-\left(\frac{\omega}{c}\right)^{2} \phi & \text { in } \Omega \\ \phi=0 & \text { on } \partial \Omega\end{cases}
$$

In the same way that (1) yields $\omega_{0 n}{ }^{s o l}$, (26) gives $\omega_{0 n}{ }^{i r r}$, parameters having no physical reality, since these modes are associated to $\mathrm{OHz}$.

In the case of perfectly conducting walls, the coefficients of (25) are easily determined:

\footnotetext{
${ }^{2}$ In fact, only one physical static mode exists among the irrotational modes found by the solver, as the stirrer presence yields a double connected frontier $(\mathrm{M}=2)$.
} 


$$
\left\{\begin{array}{l}
\alpha_{n}=\frac{1}{2 W_{n}} \frac{j \omega}{\omega^{2}-\left(\omega_{n}^{\text {sol }}\right)^{2}}\left(\vec{J}, \vec{E}_{n}^{\text {sol }}\right) \\
\beta_{n}=\frac{1}{2 W_{n}} \frac{j}{\omega}\left(\vec{J}, \vec{E}_{n}^{i r r}\right) \\
\delta_{n}=\frac{1}{2 W_{n}} \frac{j}{\omega}\left(\vec{J}, \vec{E}_{n}^{\text {stat }}\right)
\end{array}\right.
$$

where $W_{n}$ is the energy of the mode $n$.

The total field is imaginary and presents singularities at the solenoidal resonant frequencies. It is worth mentioning that the irrotational eigenvalues of (26) do not appear in these formulas: the singularities of irrotational solutions appear at $\mathrm{OHz}$ and these components are negligible near solenoidal resonant frequencies. It gives a reason to employ the common solenoidal expansion [4][20][21] as:

$$
\vec{E} \cong \sum_{n=1}^{\infty} \alpha_{n} \vec{E}_{n}^{s o l}
$$

Another interesting property is that the spectrum of an excited and closed cavity is no more discrete. To illustrate this point, we calculate the field (28), adopting from now the following: the measured point is $\mathrm{P}(1,1,1)$, and the source current $\boldsymbol{J}(1,-1,1)$ is located at source point $S(0.4,2,2)$, for coordinates given in meters and current amplitude in Amperes par square meter.

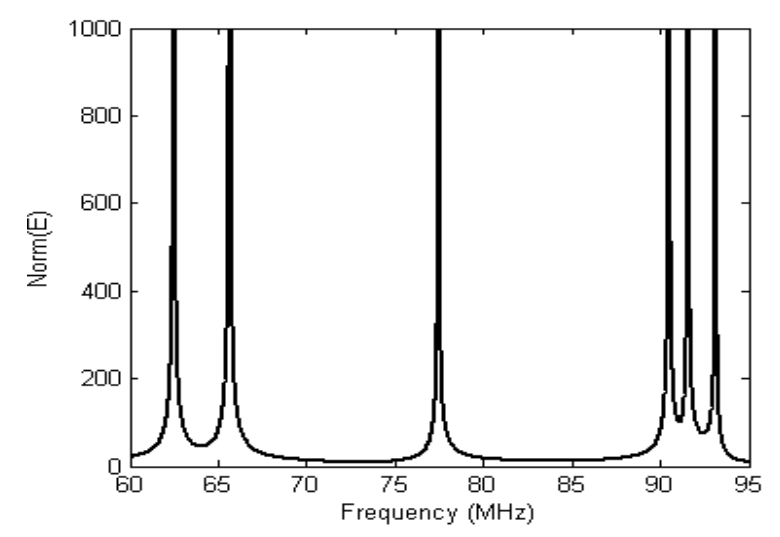

Fig. 8. $\operatorname{Norm}(E)$ at point $\mathrm{P}$ obtained by modal method without loss implementation.

As seen in Fig. 8, the spectrum of an excited lossless cavity is no more a Dirac sum and this fact has been confirmed by a direct harmonic analysis.

The modes are not only excited at resonant frequencies. When the coupling between the cavity and the internal source is taken into account, the cavity response becomes non-null between resonant frequencies. It is worth noting that in real life, this phenomenon is hidden by another one: As the RC is excited by an antenna coupled to the exterior, the RC cannot be considered as lossless.

\section{B. Losses implementation}

To suppress the frequency singularities, losses have to be modeled. A perturbation technique is employed, stating that resonant frequencies obtained in the lossless analysis (1) are substituted by complex resonant frequencies [19 ] as

$$
f_{n}^{\text {sol }}=f_{0 n}^{\text {sol }}\left(1+\frac{j}{2 Q}\right)
$$

Thus, the coefficients $\alpha_{n}$ are finite at the resonant frequencies, and for weak losses $(Q>>1)$, they are given close to the resonant frequency $f_{0 n}^{\text {sol }}$ by:

$$
\alpha_{n} \cong \frac{\frac{-Q}{f_{0 n}^{\text {sol }}}}{4 \pi W_{n}^{\text {sol }}} \frac{\frac{f}{f_{0 n}^{\text {sol }}}}{1+2 j Q \frac{f-f_{0 n}^{\text {sol }}}{f_{0 n}^{\text {sol }}}}\left(\vec{J}, \vec{E}_{n}^{\text {sol }}\right)
$$

The frequency dependent term of (30) is a narrow band approximation of a second order oscillator response.

Instead of using a mode-dependant quality factor, which would represent losses originating only from Joule effect, we consider an averaged $\mathrm{Q}$ factor that takes into account losses from various origins. The main advantage is a capability to model antenna losses that are preponderant at low frequencies. To illustrate this fact, we first evaluate the Joule losses by the following approximation [22]

$$
Q_{J}=\frac{3}{2} \frac{V}{S \delta},
$$

where $\mathrm{S}$ and $\mathrm{V}$ are the cavity surface and volume, and $\delta$ the skin depth. Leaking power by antenna can be modeled [22] by

$$
Q_{\text {Ant }}=16 \pi^{2} V\left(\frac{f}{c}\right)^{3} .
$$

Finally the composite quality factor is defined [22] by

$$
\frac{1}{Q_{C}}=\frac{1}{Q_{J}}+\frac{1}{Q_{\text {Ant }}} \text {. }
$$

The evolution of the different quality factors versus the frequency is graphed in Fig. 9. 


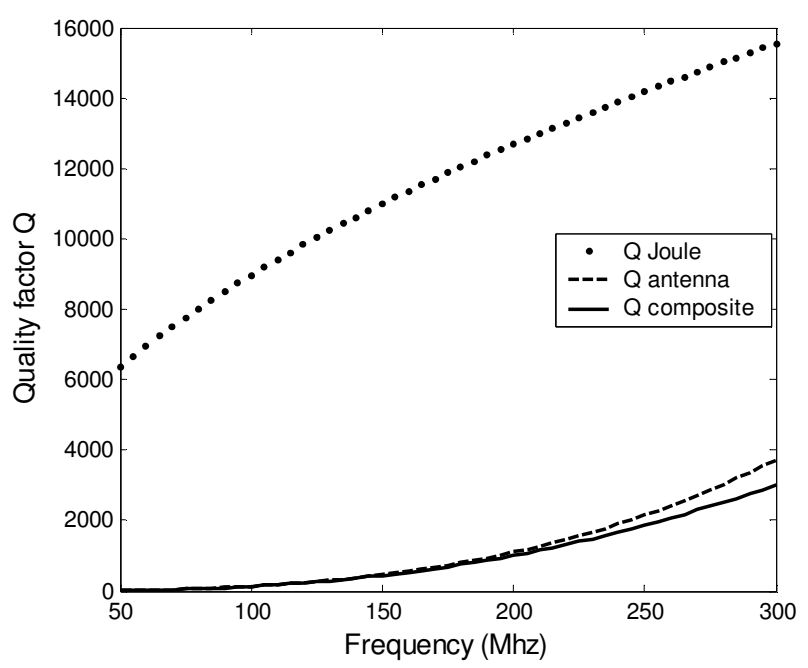

Fig. 9. Evolution of quality factor versus frequency, for the studied RC.

Figure 9 shows clearly that the Joule losses can be neglected in the very low part of the spectrum: losses are mainly due to antenna leaking. Considering two antennas (transmission and reception), $\mathrm{Q}$ is set to a low value (e.g. $\mathrm{Q}=60$ ) for the $50 \sim 100$ $\mathrm{MHz}$ band. At higher frequencies, around $260 \mathrm{MHz}, \mathrm{Q}$ is set to 1000 .

In Fig. 10 a comparison is given for the $50 \sim 100 \mathrm{MHz}$ band between the null-divergence expansion (28) and a harmonic analysis. In this later one, losses are simulated by adding surface impedances on the cavity walls, as for Joule losses. To permit a comparison between the results obtained using both methods, the surface impedance of the walls is chosen to yield a mean $Q$ factor of 60 over the frequency band.

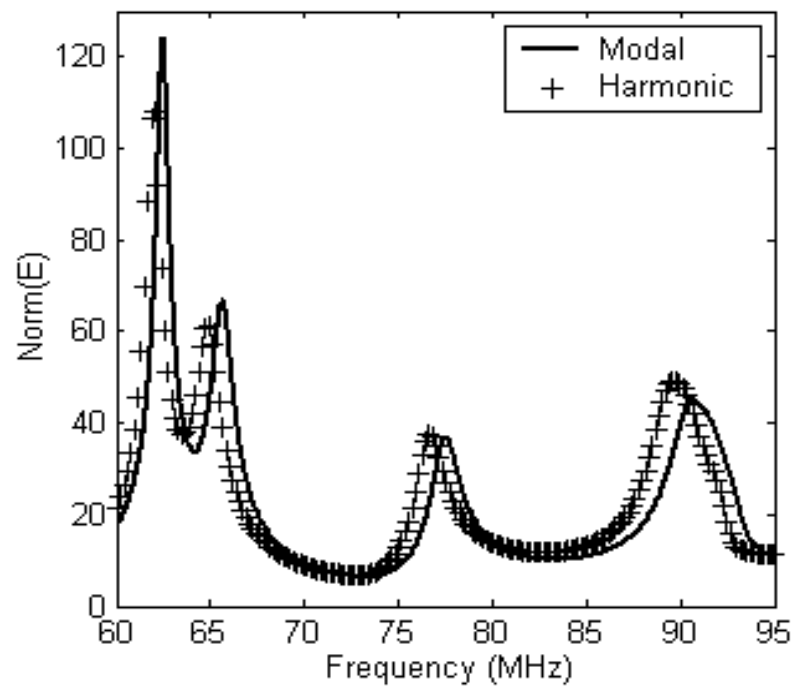

Fig. 10. $\operatorname{Norm}(\boldsymbol{E})$ at point $\mathrm{P}$ obtained by harmonic and modal methods, 50 $\sim 100 \mathrm{MHz}$ frequency band $(Q \# 60)$.

A good agreement is shown, as well as the expected $f_{0 n} / 2 Q$ resonant frequency offset [19 p. 34]. The numerous harmonic simulations necessary to approximate the resonance peaks illustrate the advantage of the modal method in matters of computational effort ${ }^{3}$.

The method is then applied to a higher frequency: 16 modes are sought around $260 \mathrm{MHz}$, and the electric field is expanded on these 16 modes using (28) and (30), with $Q=1000$. The result is plotted in Fig. 11, as well as many harmonic determinations, wherein the Surface Impedance of the walls is chosen to yield a mean $Q$ factor of 1000 over the frequency band.

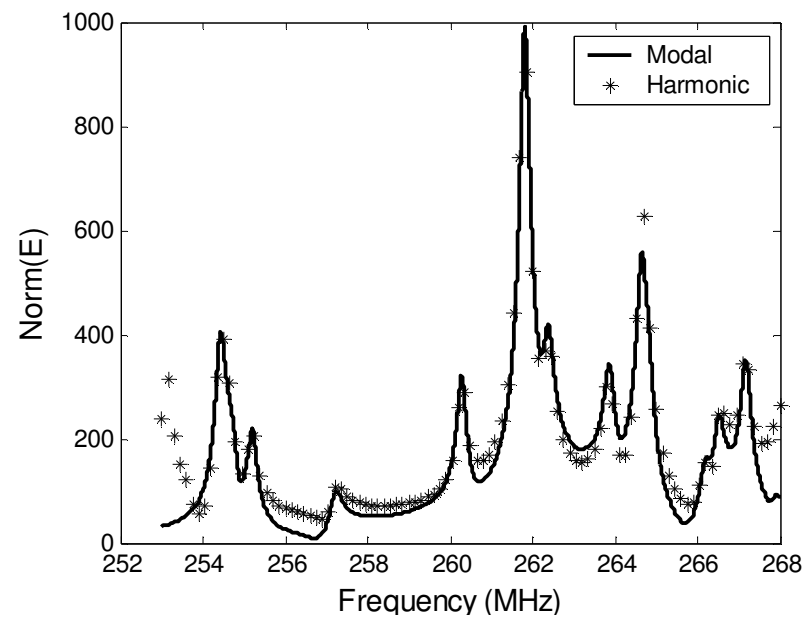

Fig. 11. $\operatorname{Norm}(\boldsymbol{E})$ at point $\mathrm{P}$ obtained by harmonic and modal method, close to $260 \mathrm{MHz}(Q=1000)$.

Besides this satisfactory comparison, two phenomena can be observed in Fig. 11. First the use of a same Q factor for all modes leads to acceptable differences for the peaks height. Secondly, the behavior at the frequency band edges gives an indication of the convergence speed of expansion (28). Figure 11 shows that the modal expansion on 16 modes is reliable in the 254 267 MHz band, thus at a $1 \mathrm{MHz}$ from the edges of the frequency band on which the eigenvalues are calculated. This $1 \mathrm{MHz}$ value corresponds to roughly $4 \mathrm{f}_{0} / \mathrm{Q} \mathrm{MHz}$, where $\mathrm{f}_{0}=$ $260 \mathrm{MHz}$.

Figures 11-12 have illustrated and validated the use of modal expansion (28): a single eigenmode determination followed by a modal expansion provides a field description in a narrow frequency band, which would need many harmonic analysis. However, one can note that (28) is only an approximation of (25), since it does not include the irrotational terms: its validity is thus reserved for measure points located far from the source point.

\section{Singularity extraction}

The source term creates a field singularity, which cannot be expanded on the only solenoidal terms (28). To illustrate the difference between (25) and (28), we conduct a harmonic analysis at $f=76.62 \mathrm{MHz}$, which corresponds to the $\mathrm{RC}$ third resonance in Fig. 10. The result is given in Fig. 12.

\footnotetext{
3 The matrices issued from harmonic analysis are complex symmetric, factorized by UMFPACK algorithm using Femlab.
} 


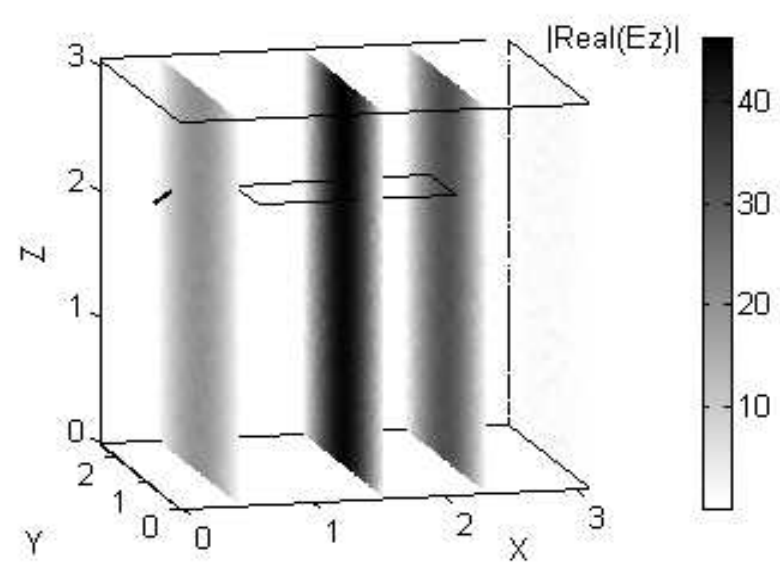

Fig. 12. Slice cartography of $\operatorname{real}(E z)$ at resonant frequency $f=76.62 \mathrm{MHz}$ obtained by harmonic analysis.

As the modes are well separated, the main term of the solenoidal expansion (28) corresponds to the third mode. $\alpha_{n=3}$ being real at the resonance, the field real component (Fig. 12) has the same pattern as $\mathrm{TM}_{110}$. In fact, a small imaginary field component does exist, due to the other modes contributions. For example, the real part of the electric field at point $\mathrm{P}$ is 36.7 and the imaginary part is 4.6. These values are deduced from both harmonic modeling and modal expansion (28). Thus (28) is a good approximation of (25) for a common interior point of the cavity, far from the antenna.

Actually, we notice on Fig. 13 a high imaginary ${ }^{4}$ field region close to the antenna, and a fast decrease of this imaginary part when the distance to the antenna increases.

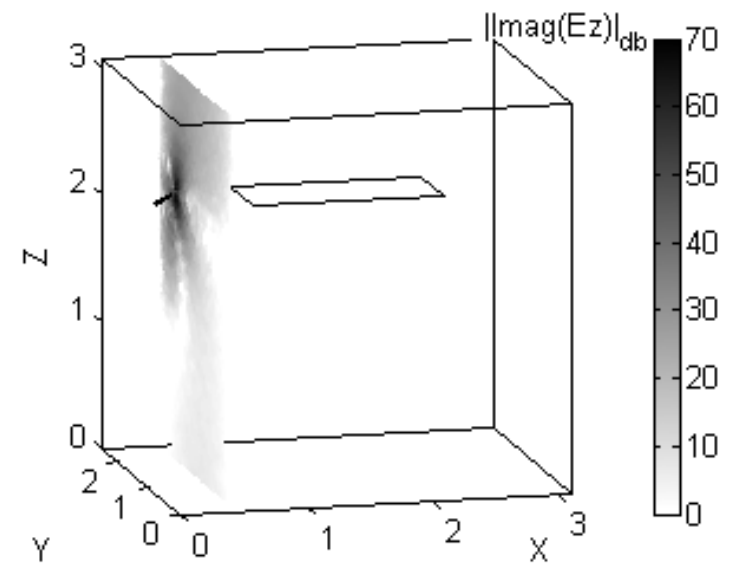

Fig. 13. Slice cartography of $20 \log [\operatorname{abs}(\operatorname{Imag}(E z))]$ at resonant frequency $\mathrm{f}=76.62 \mathrm{MHz}$, obtained by harmonic analysis (notice the E scale in $\mathrm{dB}$ ).

This phenomenon can only be explained by irrotational terms in (25). The first idea would be to expand this irrotational part on the basis of solutions of (26). For this, we discretize the scalar equation (26) by Lagrange 2 elements and portray in Fig. 14 the first irrotational mode:
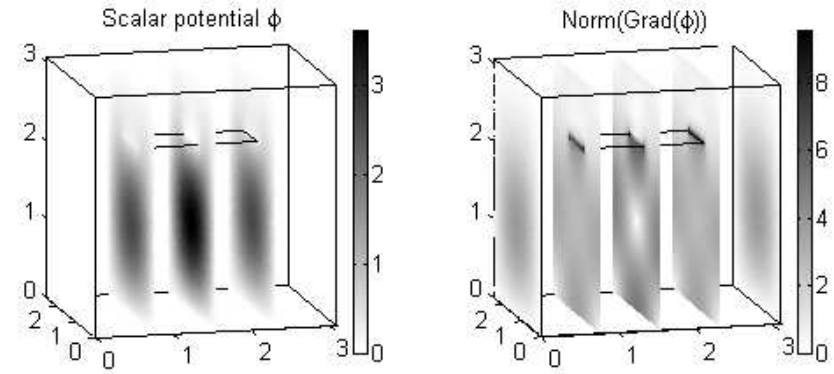

Fig. 14. Slice cartography of the first irrotational mode.

Of course, these irrotational modes do not present any singularity near the source point: this explains why the convergence is known to be very slow [19, Ac. 2.8], i.e. the number of irrotational modes to take into account in the expansion (25) is very high. In place of expanding this irrotational part of $\boldsymbol{E}$ field, we illustrate the singularity extraction technique [23] in a simple way. The Green function singularity in the RC close to the antenna is the same as the singularity of free-space Green function. On a similar way, we just suppose that the field close to the antenna corresponds to the free-space field $\boldsymbol{E}_{\boldsymbol{0}}$. This field is numerically approximated by a harmonic analysis, wherein Matched Impedance is implemented on cavity walls. Figure 15 depicts the result of the substraction of this free-space field $\boldsymbol{E}_{\boldsymbol{0}}$ to the total field observed in Fig. 13.

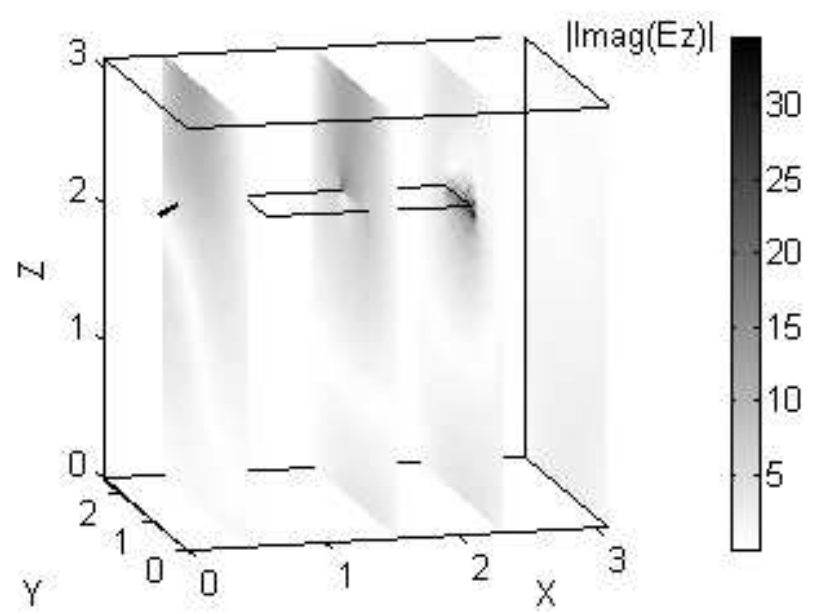

Fig. 15. Slice cartography of $\left.\mid \operatorname{Imag}\left(E z-E_{0} z\right)\right) \mid$ at resonant frequency $f=76.62$ $\mathrm{MHz}$.

As confirms Fig. 15, the field is regularized in the antenna vicinity, and can then be expanded on solenoidal modes.

\section{CONCLUSION}

In this paper a Reverberation Chamber is modeled using the Finite Element Method (FEM) modal approach. For this 3D FEM model, the large number of Degrees of Freedom implies a restriction to the lower part of the spectrum. In order to

\footnotetext{
${ }^{4}$ The cavity excitation is modeled by a current distribution
} 
analyze the RC close to the Lowest Useable Frequency (LUF), high performance solvers must be used. A comparison is given between Open Source Pyfemax and Femlab commercial code, and the issue of the null-frequency solutions is exploited to decrease the CPU time.

Second, the RC stirrer presence induces a field singularity that worsens the convergence rate: an investigation on the element kind and order proved the advantage of the second order edge elements. This modal approach is validated by a comparison to harmonic analysis, and a regularization technique is illustrated for the field in the source region.

\section{REFERENCES}

[1] J. M. Ladbury, G. H. Koepke, and D. G. Camell, "Evaluation of the NASA Langley Research Center Mode-Stirred Facility," National Institute of Standards and Technology, Boulder, CO, NIST Tech. Note $1508,1999$.

[2] C. Bruns and R. Vahldieck, "A Closer Look at Reverberation Chambers-3-D Simulation and Experimental Verification," IEEE Trans. Electromag. Compat., vol. 47, pp. 612-626, Aug. 2005.

[3] C. F. Bunting, K. J. Moeller, C. J. Reddy, and S. A. Scearce, "A TwoDimensional Finite-Element Analysis of Reverberation Chambers," IEEE Trans. Electromag. Compat., vol. 41, pp. 280-289, Nov. 1999.

[4] G. Orjubin, E. Richalot, S. Mengué, and O. Picon, "Modal Representation of a Reverberation Chamber for a Parametric Analysis of the Field Uniformity," in Proc. of EMC Zurich 2005, Topical Meeting on Reverberation Chamber, pp. 133-136, Zurich, Feb. 2005.

[5] S. Feigh, M. Clemens, R. Schuhmann, and T. Weiland, "Eigenmode Simulation of Electromagnetic Resonator Cavities with Gyrotropic Materials," IEEE Trans. Magn., vol. 40, pp. 647 - 650, Mar. 2004.

[6] P. Arbenz, R. Geus, and S. Adam, "Solving Maxvell eigenvalue problems for accelerating cavities," Phys. Rev. ST Accel. Beams, vol. 4, 022001, 2001.

[7] B. Trapp, I. Munteanu, R. Schuhmann, T. Weiland, and D. Iaon, "Eigenvalue Computation by Means of a Tree-Cotree Filtering Technique,“ IEEE Trans. Magn. vol. 38, pp. 445-448, Mar. 2002.

[8] R. Geus, "The Jacobi-Davidson algorithm for solving large sparse symmetric eigenvalue problems with application to the design of accelerator cavities," Ph.D. dissertation, Swiss Federal Institute of Technology, Zurich, Suisse, 2002.

[9] J. C. Nédélec, "Mixed finite element for IR3," Numer. Math, Vol. 35, pp 315-341, 1980.

[10] A. Bossavit, "A rationale for the "Edge-Elements" in 3-D fields computations," IEEE Trans. Magn., vol. 24, pp. 74-79, Jan. 1988.

[11] J. B. Manges and Z. J. Cendes, "A Generalized Tree-Cotree Gauge for Magnetic Field Computation”, IEEE Trans. Magn., vol. 31, pp. 1342 1347, May 1995.

[12] S. G. Perepelitsa, R. Dyczij-Edlinger, and J-F. Lee, "Finite-Element Analysis of Arbitrary Shaped Cavity Resonators Using $\mathrm{H}^{1}$ (curl) Elements," IEEE Trans. Magn., vol. 33, pp. 1776-1779, Mar. 1997.

[13] M. Salazar-Palma et al., Iterative and Self-Adaptive Finite-Elements in Electromagnetic Modeling, Artech House, London, 1998.

[14] I. Bardi, O. Biro, K. Preis, G. Vrisk, K. R. Richter, "A Comparison of the Errors Obtained with Whitney and Linear Edge Elements," IEEE Trans. Magn., vol. 33, pp. 1142-1145, Mar. 1992.

[15] D. C. Dibben and R. Metaxas, "Nodal and Edge Element Analysis of Inhomogeneously Loaded 3D Cavity," IEEE Trans. Magn., vol. 33, pp 1524-1527, Mar. 1997.

[16] M. Costabel and M. Dauge, "Weighed Regularization of Maxwell Equations in Polyhedral Domains," Numer. Math.,vol. 93, $\mathrm{n}^{\circ}$ 2, 2002.

[17] R. D. Graglia and G. Lombardi, "Singular Higher Order Complete Vector Bases for Finite Methods," IEEE Trans. Antennas Propagat., vol. 52, pp. 1672-1685, Jul. 2004.

[18] J. Van Bladel, Singular Electromagnetic Fields and Sources, Oxford Engineering Science Series, Oxford UK. Clarendon, 1991.
[19] G. Conciauro, M. Guglielmi, and R. Sorrentino, Advanced Modal Analysis. John Wiley\&Sons, New York, 1999.

[20] A. Gati, M.F. Wong, and V.F. Hanna, "New technique using poles and modes derivatives for frequency and geometry parameterization of microwave structures," 2001 IEEE MTT-S Int. Microwave Symp. Dig., vol. 2 , pp. 1019-1022, May 2001.

[21] G. Cerri, V. M. Primiani, S. Pennesi, and P. Russo, "Source Stirring Mode for Reverberation Chambers," IEEE Trans. Electromagn. Compat., Vol. 47, No. 4, pp. 815-823, Nov. 2005.

[22] D. A. Hill, M. T. Ma, A. R. Ondrejka, B. F. Riddle, M. L. Crawford, and R. T. Jonk, "Aperture excitation of electrically large, lossy cavities," IEEE Trans. Electromagn. Compat., Vol. 36, No. 3, pp. 169-177, Aug. 1994.

[23] A. Borji, S. Safavi-Naeini, "Rapid Calculation of the Green's Function in a Rectangular Enclosure With Application to Conductor Loaded Cavity Resonators," IEEE Trans. Microwave Theory Tech., vol. 52, pp. 1724-1731, Jul. 2004. 